Cochrane Database of Systematic Reviews

\title{
Adhesives for bonded molar tubes during fixed brace treatment
} (Review)

Millett DT, Mandall NA, Mattick RCR, Hickman J, Glenny AM

Millett DT, Mandall NA, Mattick RCR, Hickman J, Glenny AM.

Adhesives for bonded molar tubes during fixed brace treatment.

Cochrane Database of Systematic Reviews 2017, Issue 2. Art. No.: CD008236.

DOI: 10.1002/14651858.CD008236.pub3. 
TABLE OF CONTENTS

ABSTRACT

PLAIN LANGUAGE SUMMARY

BACKGROUN

OBJECTIVES

METHODS

RESULTS

Figure 1.

Figure 2.

DISCUSSION

AUTHORS' CONCLUSIONS

REFERENCES

CHARACTERISTICS OF STUDIES

DATA AND ANALYSES

Analysis 1.1. Comparison 1 Molar tubes versus molar bands, Outcome 1 Failure at tooth level.

Analysis 1.2. Comparison 1 Molar tubes versus molar bands, Outcome 2 Failure at participant level.

Analysis 1.3. Comparison 1 Molar tubes versus molar bands, Outcome 3 Decalcification (participant level). APPENDICES

WHAT'S NEW

HISTORY

CONTRIBUTIONS OF AUTHORS

DECLARATIONS OF INTEREST

SOURCES OF SUPPORT

NOTES

INDEX TERMS

2

3

3

4

6

6 
[Intervention Review]

\title{
Adhesives for bonded molar tubes during fixed brace treatment
}

\author{
Declan T Millett ${ }^{1}$, Nicky A Mandall 2 , Rye CR Mattick ${ }^{3}$, Joy Hickman ${ }^{4}$ Anne-Marie Glenny 5
}

1Oral Health and Development, Cork University Dental School and Hospital, Cork, Ireland. 2Orthodontic Department, Tameside General Hospital, Ashton under Lyne, UK. ${ }^{3}$ Department of Orthodontics, Newcastle Dental Hospital, Newcastle upon Tyne, UK. ${ }^{4}$ Department of Orthodontics, Glan Clwyd Hospital, Rhyl, UK. 5Division of Dentistry, School of Medical Sciences, Faculty of Biology, Medicine and Health, The University of Manchester, Manchester, UK

Contact: Declan T Millett, Oral Health and Development, Cork University Dental School and Hospital, University College, Cork, Ireland. d.millett@ucc.ie.

Editorial group: Cochrane Oral Health Group.

Publication status and date: Stable (no update expected for reasons given in 'What's new'), published in Issue 3, 2017.

Citation: Millett DT, Mandall NA, Mattick RCR, Hickman J, Glenny AM. Adhesives for bonded molar tubes during fixed brace treatment. Cochrane Database of Systematic Reviews 2017, Issue 2. Art. No.: CD008236. DOI: 10.1002/14651858.CD008236.pub3.

Copyright @ 2017 The Cochrane Collaboration. Published by John Wiley \& Sons, Ltd.

\begin{abstract}
A B S T R A C T

\section{Background}

Orthodontic treatment involves using fixed or removable appliances (dental braces) to correct the positions of teeth. The success of a fixed appliance depends partly on the metal attachments (brackets and bands) being glued to the teeth so that they do not become detached during treatment. Brackets (metal squares) are usually attached to teeth other than molars, where bands (metal rings that go round each tooth) are more commonly used. Orthodontic tubes (stainless steel tubes that allow wires to pass through them), are typically welded to bands but they may also be glued directly (bonded) to molars. Failure of brackets, bands and bonded molar tubes slows down the progress of treatment with a fixed appliance. It can also be costly in terms of clinical time, materials and time lost from education/work for the patient. This is an update of the Cochrane review first published in 2011. A new full search was conducted on 15 February 2017 but no new studies were identified. We have only updated the search methods section in this new version. The conclusions of this Cochrane review remain the same.
\end{abstract}

\section{Objectives}

To evaluate the effectiveness of the adhesives used to attach bonded molar tubes, and the relative effectiveness of the adhesives used to attach bonded molar tubes versus adhesives used to attach bands, during fixed appliance treatment, in terms of: (1) how often the tubes (or bands) come off during treatment; and (2) whether they protect the bonded (or banded) teeth against decay.

\section{Search methods}

The following electronic databases were searched: Cochrane Oral Health's Trials Register (to 15 February 2017), the Cochrane Central Register of Controlled Trials (CENTRAL; 2017, Issue 1) in the Cochrane Library (searched 15 February 2017), MEDLINE Ovid (1946 to 15 February 2017), and Embase Ovid (1980 to 15 February 2017). We searched ClinicalTrials.gov and the World Health Organization International Clinical Trials Registry Platform for ongoing trials. No restrictions were placed on the language or date of publication when searching the electronic databases.

\section{Selection criteria}

Randomised controlled trials of participants with full arch fixed orthodontic appliance(s) with molar tubes, bonded to first or second permanent molars. Trials which compared any type of adhesive used to bond molar tubes (stainless steel or titanium) with any other adhesive, were included.

Trials were also included where: 
(1) a tube was bonded to a molar tooth on one side of an arch and a band cemented to the same tooth type on the opposite side of the same arch;

(2) molar tubes had been allocated to one tooth type in one patient group and molar bands to the same tooth type in another patient group.

\section{Data collection and analysis}

The selection of papers, decision about eligibility and data extraction were carried out independently and in duplicate without blinding to the authors, adhesives used or results obtained. All disagreements were resolved by discussion.

\section{Main results}

Two trials $(n=190)$, at low risk of bias, were included in the review and both presented data on first time failure at the tooth level. Pooling of the data showed a statistically significant difference in favour of molar bands, with a hazard ratio of 2.92 ( $95 \%$ confidence intervals (Cl) 1.80 to 4.72 ). No statistically significant heterogeneity was shown between the two studies. Data on first time failure at the patient level were also available and showed statistically different difference in favour of molar bands (risk ratio $2.30 ; 95 \% \mathrm{Cl} 1.56$ to 3.41 ) (risk of event for molar tubes $=57 \%$; risk of event for molar bands $25 \%$ ).

One trial presented data on decalcification again showing a statistically significant difference in favour of molar bands. No other adverse events identified.

\section{Authors' conclusions}

From the two well-designed and low risk of bias trials included in this review it was shown that the failure of molar tubes bonded with either a chemically-cured or light-cured adhesive was considerably higher than that of molar bands cemented with glass ionomer cement. One trial indicated that there was less decalcification with molar bands cemented with glass ionomer cement than with bonded molar tubes cemented with a light-cured adhesive. However, given there are limited data for this outcome, further evidence is required to draw more robust conclusions.

\section{PLAIN LANGUAGE SUMMARY}

\section{Adhesives for bonded molar tubes during fixed brace treatment}

\section{Background}

Orthodontic treatment involves using fixed or removable dental braces to correct the positions of teeth. The success of dental braces depends partly on the metal attachments (brackets and bands) being glued to the teeth so that they do not become detached during treatment. Brackets (metal squares) are usually attached to teeth other than molars, where bands (metal rings that go round each tooth) are more commonly used. Orthodontic tubes (stainless steel tubes that allow wires to pass through them), are typically welded to bands but they may also be glued directly (bonded) to molars. Failure of brackets, bands and bonded molar tubes slows down the progress of treatment with a dental brace.

\section{Study characteristics}

The evidence in this review, which was carried out together with Cochrane Oral Health, is up-to-date as of 15 February 2017 . We included two studies that evaluated 190 participants. Both trials were conducted in the UK and both compared bonded molar tubes with molar bands.

\section{Key results}

From the limited data of two studies at low risk of bias, it would appear that bonded molar tubes are associated with a higher failure rate than with molar bands. 


\section{B A C K G R O U N D}

Orthodontic treatment involves using fixed or removable appliances (dental braces) to correct the positions of teeth. In England and Wales between April 2005 and March 2006 claims for fixed appliances were made by the General Dental Services at an approximate cost of GBP 85 million to the National Health Service (personal communication, 2009).

In the Scottish General Dental Services, the cost of fixed appliance orthodontic treatment in the 12 months prior to May 2008 was approximately GBP 8.8 million (NHS National Services Scotland 2009 , personal communication). The median adjusted cost (based on point reduction in the Index of Orthodontic Treatment Need and Complexity) of orthodontic treatment in seven European countries ranged from EUR 1120 (Lithuania) to EUR 5812 (Slovenia) (Deans 2009). In Finnish municipal health centres, the cost of orthodontic treatment per patient up to the age of 18 was, on average, FIM 7358 ( ${ }^{E}$ EUR 1237), ranging from FIM 1299 to FIM 24,751 (EUR 218 to EUR 4162, conversion rate as of 01/04/2009) (Pietila 1998). In the US, orthodontic treatment accounted for $39 \%$ of the costs ( USD 2480 +/- USD 364) of surgical-orthodontic treatment in community hospital care (Panula 2002). In the UK, orthodontic costs, on average, comprised $25 \%$ of total treatment costs for patients having combined orthodontic and surgical treatment for dentofacial deformity within the state funded National Health Service (Kumar 2006). The median orthodontic costs were EUR 1456 (interquartile range EUR 1283 to EUR 1638).

\section{Who receives orthodontic treatment?}

The majority of orthodontic treatment is carried out for children aged 10 to 14 years and is primarily concerned with correcting severe crowding and rotations, buried teeth or very prominent teeth. At age 12, 35\% were judged to have an orthodontic treatment need in the UK (Chestnutt 2006), whereas 52\% of South African children of a similar age had identifiable mal-position of the teeth (malocclusion) (Van Wyk 2005). In northeast Brazil, $77 \%$ of 13 to 15 year olds had either a moderate or severe treatment need (Marques 2007). In Spain, $23.5 \%$ of 12 year olds and $18.5 \%$ of 15 to 16 year olds had a definite treatment need (Manzanera 2009). Among adults, demand and need for orthodontics is increasing, with adults now making up between $20 \%$ to $25 \%$ of cases in US orthodontic practices (Keim 2008a).

Fixed, rather than removable, appliances produce a better treatment outcome (O'Brien 1993; Richmond 1993) and therefore are favoured by most orthodontists (Chestnutt 2006).

The success of a fixed appliance depends partly on the metal attachments (brackets and bands) being glued to the teeth so that they do not become detached during treatment. Brackets (metal squares) are usually attached to teeth other than molars, where bands (metal rings that go round each tooth) are more commonly used (Stirrups 1991). Orthodontic tubes (stainless steel tubes that allow wires to pass through them), are typically welded to bands but they may also be glued directly (bonded) to molars. In the latter case, they may be made of either stainless steel or titanium; some manufacturers produce standard and smaller sizes. The proportion of US orthodontists who routinely bond first or second molars has almost doubled in the past 6 years (Keim 2008a); with the exception of second maxillary molars, $\sim 50 \%$ of orthodontists are bonding, rather than banding, molars ( $\sim 49 \%$ maxillary first molar;
41\% maxillary second molar; 48\% mandibular first molar; $52 \%$ mandibular second molar; Keim 2008a).

Failure of brackets, bands and bonded molar tubes slows down the progress of treatment with a fixed appliance. It can also be costly in terms of clinical time, materials and time lost from education/work for the patient.

\section{Adhesives for bonding tubes to molars}

A bonded molar tube should be able to resist tensile, shear, torque and peel functional stresses if it is to remain attached to the tooth surface (Millett 1999). The adhesive should be strong enough to keep the molar tube attached to the tooth for the length of the treatment, but not so strong that the tooth surface is damaged when the tube is removed. It should also ideally be easy to use clinically, protective against dental caries (decay) and of reasonable cost.

Resin adhesives (chemically or light-cured) are routinely used for bonding brackets to anterior teeth. Available resin adhesives include 2-paste systems, no-mix adhesives and light-activated direct bonding materials (Millett 2001; Keim 2008b). Light-activated direct bonding materials may pre-coat the bracket and some are bonded via a self-etching primer rather than the conventional 2stage etch and prime method (Aljubouri 2004; Banks 2007). The use of resins for bonding of tubes to molars is more problematic than bonding brackets to anterior teeth. There is an inferior quality of etch pattern (Johnston 1998; Mattick 2000) and it is harder to maintain moisture isolation (Knoll 1986). In addition, greater biting forces, at the back rather than at the front of the mouth, may enhance molar attachment failure rates (Geiger 1983; Millett 1999), particularly of lower molar tubes (Pandis 2005; Pandis 2006).

Glass ionomer cements have previously shown weaker bond strengths than resin adhesives, although modification through the addition of resin has aimed to address this (Millett 1996). A wide variation in chemical constituents and setting reactions exists and modifications are commonly classified as resin-modified glass ionomer cements (hybrids of their resin-matrix and glass ionomer parent groups) or modified composites/compomers (resin-matrix composites with some glass ionomer filler particles) (McCabe 1998).

With the number of adhesives available, it is important to understand which group bonds tubes most reliably to molar teeth, as well as reducing or preventing dental decay during the treatment period.

This is an update of the Cochrane review first published in 2011 (Millett 2011). A new full search was conducted on 15 February 2017 but no new studies were identified. We have only updated the search methods section in this new version. The conclusions of this Cochrane review remain the same.

\section{O B JECTIVES}

To evaluate the effectiveness of the adhesives used to attach bonded molar tubes, and the relative effectiveness of the adhesives used to attach bonded molar tubes versus adhesives used to attach bands, during fixed appliance treatment, in terms of: (1) how often the tubes (or bands) come off during treatment; and (2) whether they protect the bonded (or banded) teeth against decay. 


\section{METHODS}

\section{Criteria for considering studies for this review \\ Types of studies}

Randomised controlled trials (RCTs), including those that use a split-mouth design, were included in this review.

\section{Types of participants}

Any participant with full arch fixed orthodontic appliance(s) with molar tubes, bonded to first or second permanent molars, was included. Participants who had previously undergone fixed appliance treatment have been excluded. Participants with cleft lip or palate have been excluded due to the higher prevalence of molar crossbite in this group which has been associated with a greater incidence of molar attachment failure (Hodges 2001). Those with other craniofacial syndromes, or where orthognathic surgery was required, have also been excluded.

\section{Types of interventions}

Studies which compared any type of adhesive used to bond molar tubes (stainless steel or titanium) with any other adhesive, were included. Trials were also included where:

1. a tube was bonded to a molar tooth on one side of an arch and a band cemented to the same tooth type on the opposite side of the same arch;

2. molar tubes had been allocated to one tooth type in one patient group and molar bands to the same tooth type in another patient group.

Studies have been excluded that:

1. compared adhesives from the same group, that used the same curing mechanism;

2. varied etching times;

3. bonded a molar tube to a surface other than intact buccal human enamel (e.g. to a gold, porcelain or amalgam substrate or to hypoplastic enamel);

4. used tubes of different metals or of different bonding base size or configuration on the same tooth type on opposite sides of the same arch;

5. used lip bumpers to bonded molar teeth;

6. bonded tubes to primary molars or premolars or to different molar teeth on opposite sides of the mouth;

7. did not follow participants to the end of treatment.

\section{Types of outcome measures}

The primary outcome for the review was the success of each adhesive i.e. first time bond (or band as appropriate) failure. Dichotomous data on whether the molar tube stayed cemented to the tooth or not would be recorded.

Dichotomous data on the presence or absence of decay (decalcification) associated with or around the tubes would also be recorded. If data existed on size/area of decalcifications, these would also be included.

Data on adverse events (i.e. illness, allergy, bad taste, mucosal trauma), damage to teeth on tube removal, length of treatment, treatment cost and time to replace tubes with an adhesive were recorded also.

\section{Search methods for identification of studies}

For the identification of studies included or considered for this review, detailed search strategies were developed for each database searched. These were based on the search strategy developed for MEDLINE (Ovid) but revised appropriately for each database. The search strategy used a combination of controlled vocabulary and free text terms, details of the MEDLINE search are provided in Appendix 3. Filters for identifying RCTs were not used in the search due to the low yield of studies.

\section{Electronic searches}

The following electronic databases were searched:

- Cochrane Oral Health's Trials Register (whole database, to 15 February 2017) (Appendix 1);

- the Cochrane Central Register of Controlled Trials (CENTRAL; 2017, Issue 1) in the Cochrane Library (searched 15 February 2017) (Appendix 2);

- MEDLINE Ovid (1946 to 15 February 2017) (Appendix 3);

- Embase Ovid (1980 to 15 February 2017) (Appendix 4).

No restrictions were placed on the language or date of publication when searching the electronic databases.

\section{Searching other resources}

We searched the following trial registries for ongoing studies (see Appendix 5 for details of the search strategy):

- ClinicalTrials.gov (whole database, to 15 February 2017);

- The World Health Organization International Clinical Trials Registry Platform (whole database, to 15 February 2017).

All the references lists of the included studies were checked manually to identify any additional studies.

All the first authors of trial reports were contacted in an attempt to identify any unpublished studies and clarify information about the published trials (including missing data, method of randomisation, blinding and withdrawals).

Manufacturers were contacted to confirm the cement/adhesive type and were asked about their knowledge of any unpublished or ongoing clinical trials.

Cochrane Oral Health's Trials Register and CENTRAL already contain the handsearching results for the European Journal of Orthodontics (1979 to 2007), the American Journal of Orthodontics and Dentofacial Orthopedics (1970 to 2007), the British Journal of Orthodontics (became Journal of Orthodontics in 2000) (1973 to 2008) and the Angle Orthodontist (1979 to 2007).

No additional handsearching of journals was undertaken.

\section{Data collection and analysis}

\section{Selection of studies}

The titles and abstracts (when available) of all reports identified through the searches were scanned by two review authors. Full reports were obtained for trials appearing to meet the inclusion 
criteria or for which there was insufficient information in the title and abstract to make a clear decision. All full reports were assessed for eligibility independently and in duplicate without blinding to the authors, adhesives used or results obtained. All disagreements were resolved by discussion.

A statistician was to be consulted with regard to data analysis and where doubt existed about inclusion.

\section{Data extraction and management}

Data extraction was carried out independently and in duplicate. The following data were entered on a customised data collection form.

- Date that the study was conducted.

- Year of publication.

- Treatments including details of type of adhesive used to cement molar tubes (and bands where appropriate) and type of fixed appliance used.

- Sample size by study group.

- Age of subjects.

- Number of male subjects and female subjects per study group.

- Details of withdrawals by study group.

- Outcome measures.

The primary outcome measures were first time bond failure only (relative numbers/proportion of failures per group) and decalcification in each group.

\section{Assessment of risk of bias in included studies}

Eligible trials were assessed according to the following criteria:

- generation of random sequence;

- concealed allocation of treatment;

- blinding of participants/caregivers (where feasible);

- incomplete outcome data;

- selective reporting.

A description of the domains was tabulated for each included trial, along with a judgement of low, high or unclear risk of bias as described in Chapter 8 of the Cochrane Handbook for Systematic Reviews of Interventions Version 5.1.0 (Higgins 2011).

A summary assessment of the risk of bias for the primary outcome (across domains) across studies was undertaken (Higgins 2011). Within a study, a summary assessment of low risk of bias is given when there is a low risk of bias for all key domains, unclear risk of bias when there is an unclear risk of bias for one or more key domains, and high risk of bias when there is a high risk of bias for one or more key domains. Across studies, a summary assessment is rated as low risk of bias when most information is from studies at low risk of bias, unclear risk of bias when most information is from studies at low or unclear risk of bias, and high risk of bias when the proportion of information is from studies at high risk of bias sufficient to affect the interpretation of the results.

\section{Measures of treatment effect}

For dichotomous outcomes, the estimates of effect of an intervention were expressed as risk ratios (or hazard ratio) together with $95 \%$ confidence intervals. For continuous outcomes, mean differences together with $95 \%$ confidence intervals were used.

\section{Unit of analysis issues}

This would be the participant or sites within the participant in split-mouth studies. The statistical analysis was considered inappropriate if:

1. a split-mouth design did not take the clustering of the teeth or 'pairing' into account;

2. all failures were included without taking into account multiple failures on the same tooth.

\section{Dealing with missing data}

Authors were contacted for further data where required.

\section{Assessment of heterogeneity}

Clinical heterogeneity was to be assessed by examining the participants, interventions and outcome measures included in the trials. Statistical heterogeneity was to be assessed by inspection of a graphical display of the estimated treatment effects from the trials along with their $95 \%$ confidence intervals. The significance of any discrepancies in the estimates of the treatment effects from the different trials was to be assessed by means of Cochran's test for heterogeneity and quantified by the $\mathrm{I}^{2}$ statistic.

\section{Assessment of reporting biases}

A funnel plot was to be drawn if sufficient trials (10 or more) were identified. Asymmetry of the funnel plot may indicate publication bias and other biases related to sample size, though it may also represent a true relationship between trial size and effect size. A formal investigation of the degree of asymmetry was to be undertaken following the recommendations presented in Chapter 10 of the Cochrane Handbook for Systematic Reviews of Interventions Version 5.1.0 (Higgins 2011).

If evidence of small-study effects had been identified, a sensitivity analysis exploring issues such as publication status and language of publication would have been undertaken.

\section{Data synthesis}

Comparisons were to be made firstly between any of the five main types of adhesive. If possible, comparisons were to be made within groups and, where appropriate, between chemical- and light-cured adhesives as follows:

1. chemically-cured composite (CC) - variables on composite matrix and primer;

2. light-cured composite (LC) - variables on composite matrix and primer (including self-etching primer);

3. conventional glass ionomer (GIC) - variables on powder and liquid (product is not light-cured);

4. poly-acid modified composite (Compomer) - variables on composite matrix and glass ionomer particles;

5. resin-modified glass ionomer (RMGIC) - variables on type of acid, resin and polymerisation mechanism.

Within group comparisons assessing products of different brand names to see if any adhesive of the same type performs better than another of the same type, were also to be undertaken if data allowed. For each adhesive group and between adhesive groups, comparisons were also to be undertaken between the orthognathic cases and non-orthognathic cases, if data allowed. 
Meta-analyses were to be undertaken only on studies of similar comparisons reporting the same outcome measures. Risk ratios along with 95\% confidence intervals were calculated for dichotomous data, and mean differences and 95\% confidence intervals calculated for continuous data. Data were combined using a random-effects model (fixed-effect models used if less than three studies in meta-analysis). The number needed to treat (NNT) was to be calculated to prevent one extra bonded molar tube failing, as appropriate.

\section{Sensitivity analysis}

Sensitivity analysis was to be undertaken for aspects of study quality and for potential sources of heterogeneity specified a priori as follows: excluding/including unpublished studies, excluding/ including studies of low quality and excluding/including one or more large studies to assess how much they dominate the results. In addition, analysis by angle classification, inclusion of orthognathic surgery and level of participant co-operation was to be undertaken if data allowed. The association of these factors with estimated effects was to be examined by performing randomeffects metaregression analysis in STATA version 7.0 (STATA
Corporation, USA), using the program Metareg. Further potential sources of heterogeneity were to be investigated as determined from the study reports, although these would clearly have been identified as 'post-hoc' analyses and the results treated with caution.

\section{RE S U L T S}

\section{Description of studies}

Two parallel-group randomised controlled trials (RCTs) were included (Banks 2007a; Nazir 2011). Both trials were conducted in the UK and both compared bonded molar tubes with molar bands.

\section{Risk of bias in included studies}

Both included trials were considered to be at low risk of bias (Figure 1 and Figure 2). Blinding was not included in the assessment of risk of bias for the primary outcome as it was deemed unfeasible for the comparison assessed in each trial. In addition, the primary outcome could be considered objective and not easily open to manipulation.

\section{Figure 1. Risk of bias graph: review authors' judgements about each risk of bias item presented as percentages} across all included studies.

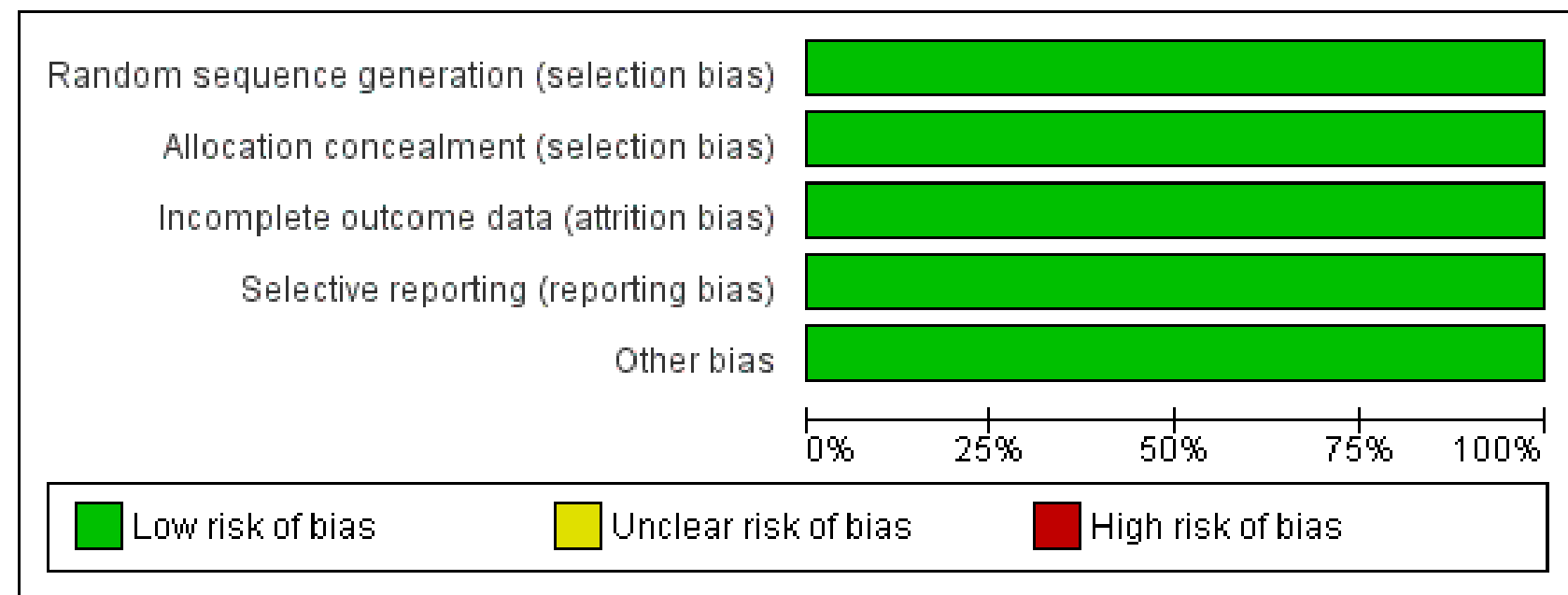


Figure 2. Risk of bias summary: review authors' judgements about each risk of bias item for each included study.

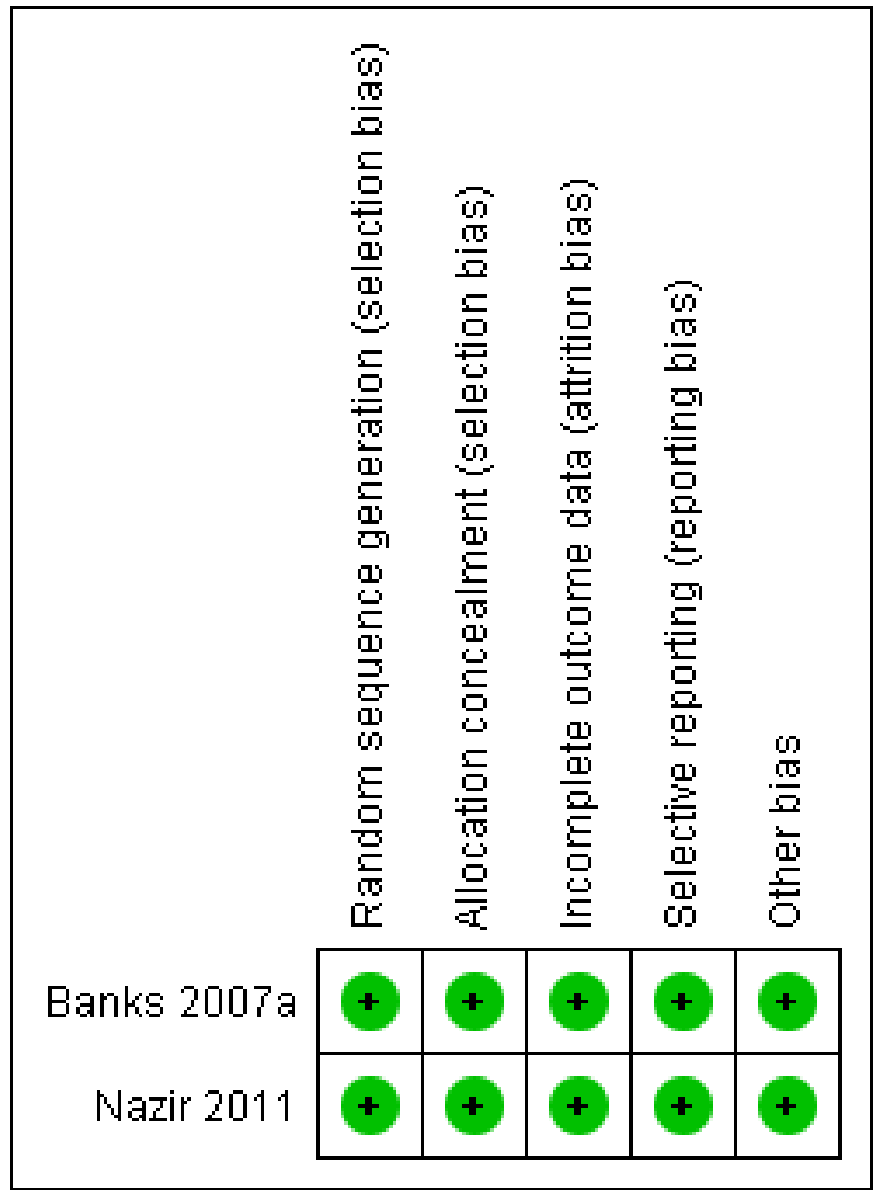

\section{Effects of interventions}

\section{Bonded molar tubes versus molar bands}

\section{First time failure}

The two included trials (Banks 2007a; Nazir 2011) both presented data on first time failure at the tooth level (Analysis 1.1). Pooling of the data showed a statistically significant difference in favour of molar bands, with a hazard ratio of 2.92 (95\% confidence interval (CI) 1.80 to 4.72 ). No statistically significant heterogeneity was shown between the two studies $\left(\mathrm{Chi}^{2}=2.36, \mathrm{df}=1(\mathrm{P}=0.12) ; \mathrm{I}^{2}=\right.$ $58 \%)$.

Only one of the trials (Banks 2007a) presented data on first time failure at the participant level; however data at participant level were available from the authors of the second trial (Nazir 2011) (Analysis 1.2). The trials showed a statistically different difference in favour of molar bands (risk ratio $2.30 ; 95 \% \mathrm{Cl} 1.56$ to 3.41 ).

Both studies made comparisons of bonded tubes or cemented bands to first permanent molars only.

\section{Decalcification}

Nazir 2011 presented data on decalcification showing a statistically significant difference in favour of molar bands (Analysis 1.3). However, given that there is currently only one trial presenting data on this outcome, further evidence is required to draw more robust conclusions.

\section{DISCUSSION}

Following application of the exclusion criteria for this review, only two relevant trials were identified. Both trials compared molar tubes versus molar bands in separate patient groups (parallel design). This, rather than a split-mouth design, has been recommended to reduce any potential bias from cross-over effects (Benson 2005; Millett 2009). Previous systematic reviews (Mandall 2003; Millett 2007) recommended that a sample size calculation be reported, that participants be followed to the end of treatment and that appropriate statistical analyses be performed. Both trials fulfilled these criteria. Future studies should continue this good practice.

\section{Failure}

The evidence with regard to a greater first time bond failure of molar tubes compared to molar bands ( $\sim 2$ to 6 times greater failure) is reported in the two trials; $33.7 \%$ versus $18.8 \%$ (Banks 2007a) and $18.4 \%$ versus $2.6 \%$ (Nazir 2011). Whether data pooling from these trials is interpreted as first time failure at tooth level or at patient level, it reveals a hazard ratio of 2.92 or 2.24 respectively in favour of molar bands. 
The different failure rates between these trials with regard to each form of molar attachment (bonded tube or band) may be partly attributed to differences in attachment bases / fitting surfaces, types of adhesives used, patient and operator factors as well as mechanics adopted.

The use of photo-etched bonding bases / band fitting surfaces to the molar tubes and bands respectively may account in part for the lower failure rates of both types of attachments in the study by Nazir 2011 compared to Banks 2007a where untreated attachments were used. Micro-etching the band fitting surface has considerably reduced band failure rates (Millett 1995; Hodges 2001) when cemented with glass ionomer, the band adhesive used in both of the included studies.

The difference in adhesives used to bond the molar tubes in each study may also have contributed to the difference in failure rates; a no-mix chemically-cured composite (Banks 2007a) and a light-cured composite (Nazir 2011). Although a previous review (Mandall 2002) suggested no statistically significant differences in failure rates of brackets bonded with either a chemically-cured or light-cured composite, the evidence was weak; the difficulty in maintaining good moisture control while a chemically-cured composite sets (7 minutes before archwire placement (Banks 2007a) compared to 40 seconds (Nazir 2011)) may account for the almost two-fold difference in bonded molar tube failure rate between these studies.

A conventional glass ionomer cement was used for band cementation in both studies (although different brands) as this is commonly used now for this purpose (Keim 2008b). Failure rates reported in both trials, with this cement type, are within the range found in previous studies (Millett 1995; Millett 2009).

Although participant's age at start of treatment has been shown to be a useful predictor of bonded molar tube survival (Millett 1999), this was not found in either trial included in this review.

Failure of bonded attachments (brackets and molar tubes) have been found to differ significantly between operators (Millett 1994; Millett 1999). Two of the three operators in the trial by Banks 2007a had a three fold higher proportion of failures than the other operator which will also account for the generally higher attachment failure rate in that trial compared to Nazir 2011. Interestingly, the latter study reports that only one band failure was found in patients, whereas multiple attachment failure episodes occurred more commonly in those with bonded molar tubes. Such details regarding attachment failure at the patient level should be reported in future trials. It may be useful also to have data regarding the overall failure of bonded brackets (per operator ideally) as this would give background data about general clinical failure rates (per operator; Banks 2007a) against which those of molar attachments (in particular bonded tubes) could be compared. Where, however, large differences exist in the numbers of subjects recruited per operator, inter-operator comparisons are of doubtful value.

The level of previous experience of each operator in bonding molar tubes before the trial commenced may also have influenced the findings. The level of previous experience of each operator with a technique-sensitive procedure such as molar bonding (Banks 2007a) should be reported in previous trials.
There are also differences in clinical protocol between these studies which should be taken into account. Distal end cutters were not used in the mouth as this was deemed likely to debond molar attachments (Nazir 2011) but this is not reported on in the trial by Banks 2007a.

As different mechanics may influence the failure of molar attachments, Mandall 2002 recommended that all patients be treated in the same manner apart from the intervention. Banks 2007a reports following a typical archwire sequence; each operator followed their own treatment sequence in the study by Nazir 2011. It is advisable that future trials standardize and report on the archwire sequence adopted. Glass ionomer bite planes were used initially where necessary to reduce the effect of occlusal stress on bonded molar tubes in the Banks 2007a trial whereas patients deemed likely to debond molar tubes due to the occlusion were excluded by Nazir 2011. While assessing for occlusal interferences that may affect bond failure was recommended in a previous review (Mandall 2002), this may have introduced exclusion bias in the Nazir 2011 trial and may have helped account for the lower failure rate of bonded molar attachments reported compared to Banks 2007a. Additionally, second permanent molars were not included unless required for overbite reduction or their alignment (Banks 2007a) whereas this is not reported by Nazir 2011.

\section{Decalcification}

The trial by Nazir 2011 found more patients experienced decalcification on teeth with molar tubes (64\%) compared to those with molar bands (36\%), although these enamel changes were minor using the index of assessment and were not seen in the wet state. The evidence from a previous review was insufficient with regard to glass ionomer cement for prevention of enamel decalcification on banded first permanent molars (Millett 2009). Furthermore Benson 2005 identified weak evidence for greater effectiveness of glass ionomer based adhesives over conventional composite resins for prevention of decalcification around bonded brackets.

\section{Should molar tubes continue to be used in clinical practice?}

Within the limitations of the evidence presented in this review from two trials, it would appear that if bonded molar tubes are to be used, a higher failure rate and more decalcification is likely to be anticipated than with molar bands. Both trials used a range of experienced personnel and were undertaken in hospital and specialist practice settings (Banks 2007a; Nazir 2011), the latter study in both settings. The findings, therefore, indicate the effectiveness of the molar tubes/bands used in these settings; it should be realised that the findings are specific to the types of molar attachments / adhesives used and the operator experience / proficiency with each procedure. External validity of the findings is, therefore, limited. Further trials with other designs of molar tube / adhesive systems may yield different results.

\section{AUTHORS' CONCLUSIONS}

\section{Implications for practice}

From two well-designed and low risk of bias trials included in this review. 
The failure of molar tubes bonded with either a chemically-cured or light-cured adhesive was considerably higher than that of molar bands cemented with glass ionomer cement. One trial indicated that there was less decalcification with molar bands cemented with glass ionomer cement than with bonded molar tubes cemented with a light-cured adhesive.

\section{Implications for research}

Further similarly well-designed trials, taking into account recommendations as outlined above, are required using different adhesive systems / molar tube design. 


\section{RE F E R E N C E S}

\section{References to studies included in this review}

Banks 2007a \{published data only\}

Banks P, Macfarlane TV. Bonded versus banded first molar attachments: a randomized controlled clinical trial. Journal of Orthodontics 2007;34(2):128-36.

\section{Nazir 2011 \{published and unpublished data\}}

Nazir M, Walsh T, Mandall NA, Matthew S, Fox D. Banding versus bonding of first permanent molars: a multi-centre randomized controlled trial. Journal of Orthodontics 2011;38(2):81-9. [DOI: 10.1179/14653121141308]

\section{Additional references}

\section{Aljubouri 2004}

Aljubouri YD, Millett DT, Gilmour WH. Six and 12 months' evaluation of a self-etching primer versus two-stage etch and prime for orthodontic bonding: a randomized clinical trial. European Journal of Orthodontics 2004;26(6):565-71.

\section{Banks 2007}

Banks P, Thiruvenkatachari B. Long-term evaluation of bracket failure with a self-etching primer: a randomized controlled trial. Journal of Orthodontics 2007;34(4):243-51.

\section{Benson 2005}

Benson PE, Shah AA, Millett DT, Dyer F, Parkin N, Vine RS. Fluorides, orthodontics and demineralization: a systematic review. Journal of Orthodontics 2005;32(2):102-14.

\section{Chestnutt 2006}

Chestnutt IG, Burden DJ, Steele JG, Pitts NB, Nuttall NM, Morris AJ. The orthodontic condition of children in the United Kingdom, 2003. British Dental Journal 2006;200(11):609-12.

\section{Deans 2009}

Deans J, Playle R, Durning P, Richmond S. An exploratory study of the cost-effectiveness of orthodontic care in seven European countries. European Journal of Orthodontics 2009;31(1):90-4.

\section{Geiger 1983}

Geiger AM, Gorelick L, Gwinnett AJ. Bond failure rates of facial and lingual attachments. Journal of Clinical Orthodontics 1983;17(3):165-9.

\section{Higgins 2011}

Higgins JPT, Green S (editors). Cochrane Handbook for Systematic Reviews of Interventions Version 5.1.0 [updated March 2011]. The Cochrane Collaboration, 2011. Available from www.cochrane-handbook.org.

\section{Hodges 2001}

Hodges SJ, Gilthorpe MS, Hunt NP. The effect of micro-etching on the retention of orthodontic molar bands: a clinical trial. European Journal of Orthodontics 2001;23(1):91-7.

\section{Johnston 1998}

Johnston CD, Burden DJ, Hussey DL, Mitchell CA. Bonding to molars: the effect of etch time (an in vitro study). European Journal of Orthodontics 1998;20(2):195-9.

\section{Keim 2008a}

Keim RG, Gottlieb EL, Nelson AH, Vogels DS 3rd. 2008 JCO study of orthodontic diagnosis and treatment procedures, part 1: results and trends. Journal of Clinical Orthodontics 2008;42(11):625-40.

\section{Keim 2008b}

Keim RG, Gottlieb EL, Nelson AH, Vogels DS 3rd. 2008 JCO study of orthodontic diagnosis and treatment procedures. Part 2: breakdown of selected variables. Journal of Clinical Orthodontics 2008;42(12):699-710.

\section{Knoll 1986}

Knoll M, Gwinnett AJ, Wolff MS. Shear strength of brackets bonded to anterior and posterior teeth. American Journal of Orthodontics 1986;89(6):476-9.

\section{Kumar 2006}

Kumar S, Williams AC, Sandy JR. Orthognathic treatment: how much does it cost?. European Journal of Orthodontics 2006;28(6):520-8.

\section{Mandall 2002}

Mandall NA, Millett DT, Mattick CR, Hickman J, Worthington HV, Macfarlane TV. Orthodontic adhesives: a systematic review. Journal of Orthodontics 2002;29(3):205-10.

\section{Mandall 2003}

Mandall NA, Hickman J, Macfarlane TV, Mattick RCR, Millett DT, Worthington HV. Adhesives for fixed orthodontic brackets. Cochrane Database of Systematic Reviews 2003, Issue 2. [DOI: 10.1002/14651858.CD002282]

\section{Manzanera 2009}

Manzanera D, Monteil-Company JM, Almerich-Silla JM, Gandia JL. Orthodontic treatment need in Spanish schoolchildren: an epidemiological study using the Index of Orthodontic Treatment Need. European Journal of Orthodontics 2009;31(2):180-3.

\section{Marques 2007}

Marques CR, Couto GB, Orestes Cardoso S. Assessment of orthodontic treatment needs in Brazilian schoolchildren according to the Dental Aesthetic Index (DAI). Community Dental Health 2007;24(3):145-8.

\section{Mattick 2000}

Mattick CR, Hobson RS. A comparative micro-topographic study off the buccal enamel of different tooth types. Journal of Orthodontics 2000;27(2):143-8.

\section{McCabe 1998}

McCabe JF. Resin-modified glass-ionomers. Biomaterials 1998;19(6):521-7. 


\section{Millett 1994}

Millett DT, Gordon PH. A 5-year clinical review of bond failure with a no-mix adhesive (Right on). European Journal of Orthodontics 1994;16(3):203-11.

\section{Millett 1995}

Millett DT, McCabe JF, Bennett TG, Carter NE, Gordon PH. The effect of sandblasting on the retention of first molar orthodontic bands cemented with glass ionomer cement. British Journal of Orthodontics 1995;22(2):161-9.

\section{Millett 1996}

Millett DT, McCabe. Orthodontic bonding with glass ionomer cements: a review. European Journal of Orthodontics 1996;18(4):385-99.

\section{Millett 1999}

Millett DT, Hallgren A, Fornell AC, Robertson M. Bonded molar tubes: a retrospective evaluation of clinical performance. American Journal of Orthodontics and Dentofacial Orthopedics 1999;115(6):667-74.

\section{Millett 2001}

Millett DT, Letters S, Roger E, Cummings A, Love J. Bonded molar tubes: an in vitro evaluation. Angle Orthodontist 2001;71(5):380-5.

\section{Millett 2007}

Millett DT, Glenny AM, Mattick RCR, Hickman J, Mandall NA. Adhesives for fixed orthodontic bands. Cochrane Database of Systematic Reviews 2007, Issue 2. [DOI: 10.1002/14651858.CD004485.pub3]

\section{Millett 2009}

Millett D, Mandall N, Hickman J, Mattick R, Glenny AM. Adhesives for fixed orthodontic bands. A systematic review. Angle Orthodontist 2009;79(1):193-9.

\section{O'Brien 1993}

O'Brien KD, Shaw WC, Roberts CT. The use of occlusal indices in assessing the provision of orthodontic treatment by the hospital orthodontic service of England and Wales. British Journal of Orthodontics 1993;20(1):25-35.

\section{Pandis 2005}

Pandis N, Christensen L, Eliades T. Long-term clinical failure rate of molar tubes with a self-etching primer. Angle Orthodontist 2005;75(6):1000-2.

\section{Pandis 2006}

Pandis N, Polychronopoulou A, Eliades T. A comparative assessment of the failure rate of molar tubes bonded with a self-etching primer and conventional acid-etching. World Journal of Orthodontics 2006;7(1):41-4.

\section{Panula 2002}

Panula K, Keski-Nisula L, Keski-Nisula K, Oikarinen K, KeskiNisula S. Costs of surgical-orthodontic treatment in community hospital care: an analysis of the different phases of treatment. International Journal of Adult Orthodontics and Orthognathic Surgery 2002;17(4):297-306.

\section{Pietila 1998}

Pietila T, Sintonen H, Pietila I, Widstrom E, Varrela J, Alanen P. Cost and productivity analysis of orthodontic care in Finland. Community Dentistry and Oral Epidemiology 1998;26(4):283-8.

\section{Richmond 1993}

Richmond S, Shaw WC, Stephens CD, Webb WG, Roberts CT, Andrews M. Orthodontics in the general dental service of England and Wales: a critical assessment of standards. British Dental Journal 1993;174(9):315-29.

\section{Stirrups 1991}

Stirrups DR. A comparative clinical trial of a glass ionomer and a zinc phosphate cement for securing orthodontic bands. British Journal of Orthodontics 1991;18(1):15-20.

\section{Van Wyk 2005}

Van Wyk PJ, Drummond RJ. Orthodontic status and treatment need of 12-year-old children in South Africa using the Dental Aesthetic Index. Journal of the South African Dental Association 2005;60(8):334-6, 338.

\section{References to other published versions of this review Millett 2011 \\ Millett DT, Mandall NA, Mattick RCR, Hickman J, Glenny AM. Adhesives for bonded molar tubes during fixed brace treatment. Cochrane Database of Systematic Reviews 2011, Issue 6. [DOI: 10.1002/14651858.CD008236.pub2]}

\section{CHARACTERISTICS OF STUDIES}

Characteristics of included studies [ordered by study ID]

Banks 2007a

Methods $\quad$ RCT, parallel group.

Multicentre: 2 UK hospital orthodontic clinics.

Follow-up: end or discontinuation of treatment. 
Banks 2007a (Continued)

\section{Age: 9 to 33 years.}

Duration of treatment: 7 months to 41 months.

Interventions Group 1. Single first molar tubes bonded with a no-mix chemically cured composite (Rely-A-Bond) after a 30 second etch (55 participants; 181 molar tubes).

Group 2. Non-sandblasted bands cemented with conventional glass ionomer cement (Intact) (55 participants; 186 bands).

All participants received similar straight wire mechanics and archwire sequences.

\section{Outcomes}

First time failure (detachment or loosening of the attachment). The primary outcome was attachment failure (tooth level) and secondary outcome the number of failures per patient (participant level).

Time to failure.

Adhesive Remnant Index.

\section{Notes}

\section{Risk of bias}

\begin{tabular}{lll}
\hline Bias & Authors' judgement & Support for judgement \\
\hline $\begin{array}{l}\text { Random sequence genera- } \\
\text { tion (selection bias) }\end{array}$ & Low risk & Quote: "random number tables". \\
\hline $\begin{array}{l}\text { Allocation concealment } \\
\text { (selection bias) }\end{array}$ & Low risk & $\begin{array}{l}\text { Quote: "Each operator enrolled participants and assigned them to their group } \\
\text { using their sealed [opaque] envelopes, which blinded the operator and partici- } \\
\text { pant to the assignment before enrolment". }\end{array}$ \\
\hline
\end{tabular}

Incomplete outcome data Low risk Comment: all participants accounted for.

(attrition bias)

All outcomes

\begin{tabular}{lll}
\hline $\begin{array}{l}\text { Selective reporting (re- } \\
\text { porting bias) }\end{array}$ & Low risk & Comment: all relevant outcomes reported. \\
\hline Other bias & Low risk & Comment: no other bias evident. \\
\hline
\end{tabular}

\section{Nazir 2011}

\begin{tabular}{ll}
\hline Methods & RCT, parallel group. \\
Multicentre: 3 UK orthodontic clinics. \\
Follow-up: end of treatment. \\
\hline
\end{tabular}

Participants 80 patients starting upper and lower fixed appliance (pre-adjusted edgewise) treatment.

Age: 10 to 18 years.

Duration of treatment: unclear.

Interventions

Group 1. Tubes bonded with light-cured composite (3M Unitek Transbond XT) to all 4 first permanent molar teeth for each participant (38 participants analysed; 152 tubes). 
Nazir 2011 (Continued)

Group 2. Bands cemented with glass ionomer (3M ESPE Ketac-Cem) to all 4 first permanent molar teeth for each participant (38 participants analysed; 152 bands).

\begin{tabular}{ll}
\hline Outcomes & First time failure. \\
& Decalcification.
\end{tabular}

Decalcification.

\section{Notes}

\section{Risk of bias}

\begin{tabular}{|c|c|c|}
\hline Bias & Authors' judgement & Support for judgement \\
\hline $\begin{array}{l}\text { Random sequence genera- } \\
\text { tion (selection bias) }\end{array}$ & Low risk & Quote: "random number tables". \\
\hline $\begin{array}{l}\text { Allocation concealment } \\
\text { (selection bias) }\end{array}$ & Low risk & $\begin{array}{l}\text { Quote: "allocations were concealed in envelopes marked with each subject's } \\
\text { identification number and held in a central place. The operator and patient re- } \\
\text { mained blind to the attachment type until after the consent and registration } \\
\text { procedures". }\end{array}$ \\
\hline $\begin{array}{l}\text { Incomplete outcome data } \\
\text { (attrition bias) } \\
\text { All outcomes }\end{array}$ & Low risk & $\begin{array}{l}\text { Comment: all participants accounted for ( } 80 \text { randomised; } 76 \text { analysed for fail- } \\
\text { ure; } 74 \text { analysed for decalcification). }\end{array}$ \\
\hline $\begin{array}{l}\text { Selective reporting (re- } \\
\text { porting bias) }\end{array}$ & Low risk & Comment: all relevant outcomes reported. \\
\hline Other bias & Low risk & Comment: no other bias evident. \\
\hline
\end{tabular}

$\mathrm{RCT}=$ randomised controlled trial.

\section{DATA AND ANALYSES}

\section{Comparison 1. Molar tubes versus molar bands}

\begin{tabular}{llllll}
\hline Outcome or subgroup title & No. of studies & $\begin{array}{l}\text { No. of partici- } \\
\text { pants }\end{array}$ & Statistical method & Effect size \\
\hline 1 Failure at tooth level & 2 & & Hazard Ratio (Fixed, 95\% Cl) & $2.92[1.80,4.72]$ \\
\hline 2 Failure at participant level & 2 & 186 & Risk Ratio (M-H, Fixed, 95\% Cl) & $2.30[1.56,3.41]$ \\
\hline 3 Decalcification (participant level) & 1 & 74 & $\begin{array}{l}\text { Risk Ratio (M-H, Random, 95\% } \\
\text { Cl) }\end{array}$ & $1.85[1.22,2.79]$ \\
\hline
\end{tabular}


Analysis 1.1. Comparison 1 Molar tubes versus molar bands, Outcome 1 Failure at tooth level.

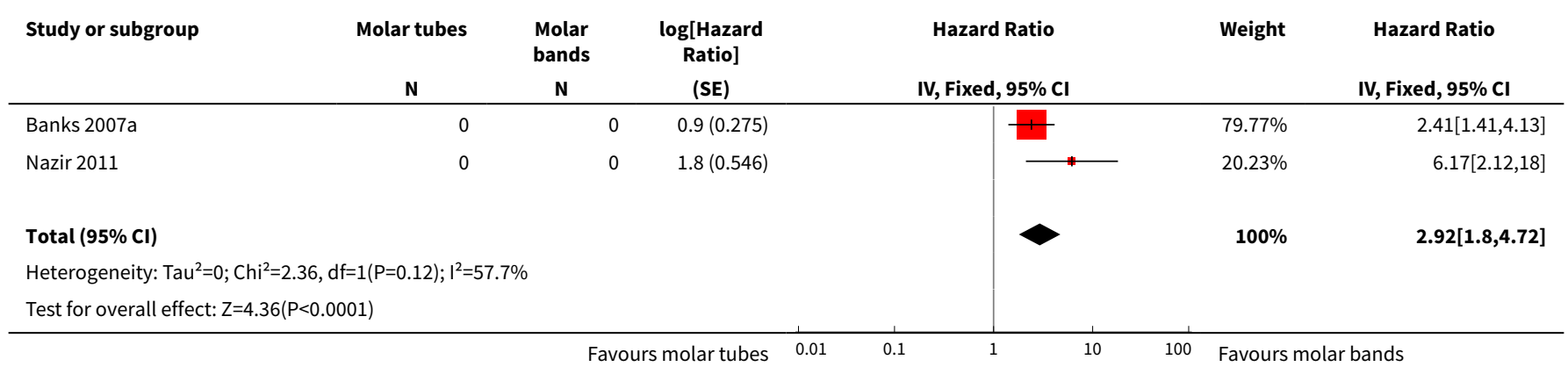

Analysis 1.2. Comparison 1 Molar tubes versus molar bands, Outcome 2 Failure at participant level.

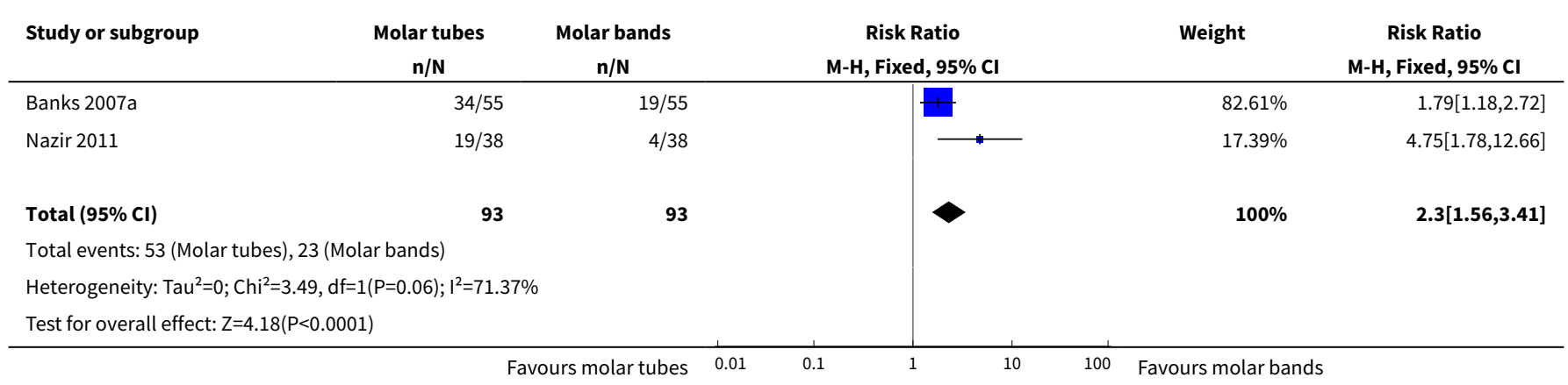

Analysis 1.3. Comparison 1 Molar tubes versus molar bands, Outcome 3 Decalcification (participant level).

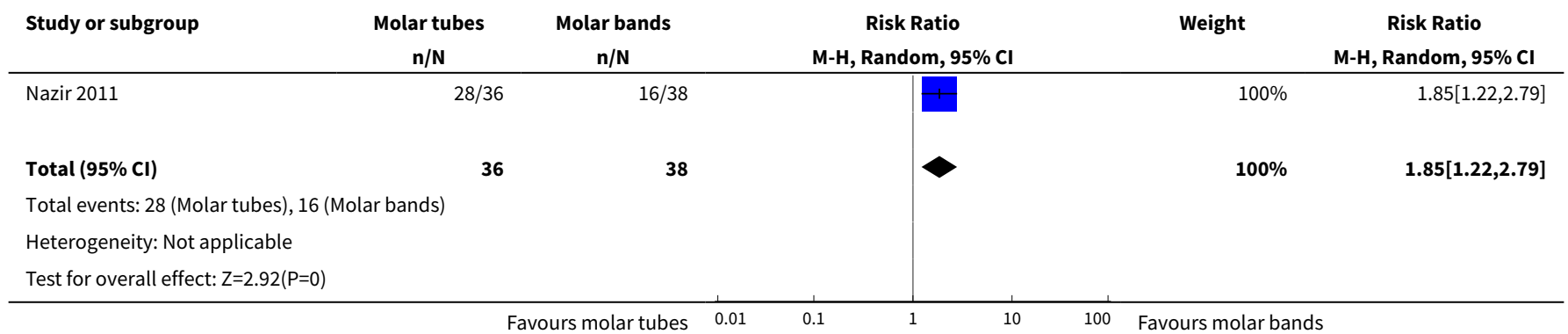

\section{APPENDICES}

\section{Appendix 1. Cochrane Oral Health's Trials Register search strategy}

From May 2016, searches of the Cochrane Oral Health's Trials Register were undertaken using the Cochrane Register of Studies and the search strategy below:

((molar ${ }^{\star}$ and tube $\left.{ }^{\star}\right):$ ti,ab) AND (INREGISTER)

Previous searches of the Cochrane Oral Health's Trials Register were undertaken in March 2009, September 2010 and December 2010 , using the Procite software and the search strategy below: 
$\left(\right.$ molar $^{\star}$ AND tube $\left.{ }^{\star}\right)$

\section{Appendix 2. Cochrane Central Register of Controlled Clinical Trials (CENTRAL) search strategy}

$\# 1$ exp Orthodontics

\#2 orthodontic*

\#3 molar* near/3 tube*

\#4 (\#1 or \#2) and \#3

\section{Appendix 3. MEDLINE (Ovid) search strategy}

1. exp Orthodontics/

2. orthodontic\$.mp.

3. (molar\$ adj3 tube\$).mp.

4. (1 or 2$)$ and 3

5. exp Composite Resins/

6. exp Glass Ionomer Cements/

7. Resin Cements/

8. exp Dental Bonding/

9. (resin\$ or cement or bond\$ or "polyacid-modified composite resin\$" or compomer\$ or composite\$ or glass-ionomer\$ or "glass ionomer

\$" or adhesive\$ or "self-etching primer\$" or "self etching primer\$")

10. or $/ 5-9$

11.4 and 10

\section{Appendix 4. Embase (Ovid) search strategy}

1. exp Orthodontics/

2. orthodontic\$.mp.

3. (molar\$ adj3 tube\$).mp.

4. (1 or 2 ) and 3

5. $\exp$ Composite Resins/

6. exp Glass Ionomer Cements/

7. Resin Cements/

8. exp Dental Bonding/

9. (resin\$ or cement $\$$ or bond\$ or "polyacid-modified composite resin\$" or compomer\$ or composite\$ or glass-ionomer\$ or "glass ionomer $\$$ " or adhesive or "self-etching primer\$" or "self etching primer\$")

10. or/5-9

11.4 and 10

\section{Appendix 5. ClinicalTrials.gov and the WHO International Clinical Trials Registry search strategy}

adhesive and molar and tube

WHAT'S NEW

\begin{tabular}{lll}
\hline Date & Event & Description \\
\hline 6 March 2017 & Review declared as stable & $\begin{array}{l}\text { This review will not be updated until a substantial body of evi- } \\
\text { dence on the topic becomes available. If trials are conducted and } \\
\text { found eligible for inclusion in the future, the review would then } \\
\text { be updated accordingly. }\end{array}$ \\
\hline
\end{tabular}

\section{H ISTORY}

Protocol first published: Issue 1, 2010

Review first published: Issue 6, 2011 


\begin{tabular}{lll}
\hline Date & Event & Description \\
\hline 15 February 2017 & $\begin{array}{l}\text { New citation required but conclusions } \\
\text { have not changed }\end{array}$ & $\begin{array}{l}\text { New search, no new studies identified. Only search methods sec- } \\
\text { tions updated. Minor edits. }\end{array}$ \\
\hline 15 February 2017 & New search has been performed & $\begin{array}{l}\text { An update search of all databases was conducted 15th February } \\
\text { 2017. No additional studies were identified. }\end{array}$ \\
\hline
\end{tabular}

\section{CONTRIBUTIONS OFAUTHORS}

Declan Millett was responsible for the conception of the review.

The first draft of the review was written by Declan Millett and Anne-Marie Glenny, with comments from Joy Hickman, Rye Mattick and Nicky Mandall.

\section{DECLARATIONS OF INTEREST}

Declan T Millett: none known. Nicky A Mandall: none known. Rye CR Mattick: none known. Joy Hickman: none known. Anne-Marie Glenny: none known. Anne-Marie Glenny is an editor with Cochrane Oral Health.

\section{SOURCES OF SUPPORT}

\section{Internal sources}

- University Dental School and Hospital, Wilton, Cork, Ireland.

- School of Dentistry, The University of Manchester, Manchester, UK.

- Newcastle Dental Hospital, Newcastle upon Tyne, UK.

- Glan Clwyd Hospital, Rhyl, UK.

\section{External sources}

- National Institute for Health Research (NIHR), UK.

This project was supported by the NIHR, via Cochrane Infrastructure funding to Cochrane Oral Health. The views and opinions expressed herein are those of the review authors and do not necessarily reflect those of the Systematic Reviews Programme, the NIHR, the NHS or the Department of Health.

- Cochrane Oral Health Global Alliance, Other.

The production of Cochrane Oral Health reviews has been supported financially by our Global Alliance since 2011 (oralhealth.cochrane.org/partnerships-alliances). Contributors over the past year have been the British Association for the Study of Community Dentistry, UK; the British Society of Paediatric Dentistry, UK; the Canadian Dental Hygienists Association, Canada; the Centre for Dental Education and Research at All India Institute of Medical Sciences, India; the National Center for Dental Hygiene Research \& Practice, USA; New York University College of Dentistry, USA; and NHS Education for Scotland, UK.

\section{NOTES}

This review will not be updated until a substantial body of evidence on the topic becomes available. If trials are conducted and found eligible for inclusion in the future, the review would then be updated accordingly.

\section{IN DEX TERMS}

\section{Medical Subject Headings (MeSH)}

*Light-Curing of Dental Adhesives; *Orthodontic Brackets; *Self-Curing of Dental Resins; Dental Cements [ ${ }^{\star}$ standards]; Dental Restoration Failure; Molar; Randomized Controlled Trials as Topic

\section{MeSH check words}

Humans 